\title{
MAGNIMS consensus guidelines on the use of MRI in multiple sclerosis-establishing disease prognosis and monitoring patients
}

\author{
Mike P. Wattjes, Àlex Rovira, David Miller, Tarek A. Yousry, Maria P. Sormani, Nicola de Stefano, \\ Mar Tintoré, Cristina Auger, Carmen Tur, Massimo Filippi, Maria A. Rocca, Franz Fazekas, Ludwig Kappos, \\ Chris Polman, Frederik Barkhof and Xavier Montalban on behalf of the MAGNIMS study group
}

MS Centre Amsterdam Department of Radiology \& Nuclear Medicine and Neurology, VU University Medical Centre, De Boelelaan 1117, $1081 \mathrm{HV}$ Amsterdam, Netherlands (M.P.W. C.P., F.B.). Magnetic Resonance Unit (À.R. C.A., X.M.), Neurology/ Neuroimmunology Unit (M.T., C.T.), Cemcat, Hospital Vall d'Hebron, Autonomous University of Barcelona, Spain. NMR Research Unit, Queen Square MS

Centre (D.M.), Lysholm Department of Neuroradiology, UCLH National Hospital for Neurology and Neurosurgery (T.A.Y.), University College London Institute of Neurology, UK Biostatistics Unit, Department of Health Sciences, University of Genoa, Italy (M.P.S.)

Department of Neurological and Behavioural Sciences, University of Siena,

Italy (N.d.S.).

Neuroimaging

Research Unit, Institute of Experimental

Neurology, Division of Neuroscience, San

Raffaele Scientific

Institute, Vita-Salute

San Raffaele University,

Italy (M.F., M.A.R.).

Department of

Neurology, Medical

University of Graz,

Austria (F.F.).

Department of

Neurology, University

of Basel, Switzerland

(L.K.)

Correspondence to:

M.P.W.

m.wattjes@vumc.nl

\begin{abstract}
The role of MRI in the assessment of multiple sclerosis (MS) goes far beyond the diagnostic process. MRI techniques can be used as regular monitoring to help stage patients with MS and measure disease progression. MRI can also be used to measure lesion burden, thus providing useful information for the prediction of long-term disability. With the introduction of a new generation of immunomodulatory and/or immunosuppressive drugs for the treatment of MS, MRI also makes an important contribution to the monitoring of treatment, and can be used to determine baseline tissue damage and detect subsequent repair. This use of MRI can help predict treatment response and assess the efficacy and safety of new therapies. In the second part of the MAGNIMS (Magnetic Resonance Imaging in MS) network's guidelines on the use of $\mathrm{MRI}$ in MS, we focus on the implementation of this technique in prognostic and monitoring tasks. We present recommendations on how and when to use MRI for disease monitoring, and discuss some promising MRI approaches that may be introduced into clinical practice in the near future.
\end{abstract}

Wattjes, M. P. et al. Nat. Rev. Neurol. 11, 597-606 (2015); published online 15 September 2015; doi:10.1038/nrneurol.2015.157

\section{Introduction}

The valuable contribution of MRI to the diagnosis, and particularly the differential diagnosis, of multiple sclerosis (MS) has been highlighted in many review articles and position papers. ${ }^{1-3}$ Additionally, the use of MRI for MS diagnosis has accelerated since the introduction and subsequent revisions of the International Panel criteria for the diagnosis of MS (also called the McDonald criteria). ${ }^{4-6}$ Nonetheless, the use of MRI in follow-up monitoring of MS disease activity has been somewhat overlooked, despite the fact that this technique offers promising prospects for patient care.

The potential for MRI measures to facilitate the assessment and monitoring of treatment efficacy is well recognized. With the approval of a new generation of MS drugs, the applications of MRI in treatment monitoring have broadened beyond tracking of disease progression to include detection of opportunistic infections and paradoxical reactions. The emerging pharmacological approaches that target pathogenetic pathways for preventing MS progression (for example, by promoting remyelination) will require new imaging approaches to monitor disease activity. ${ }^{7-9}$

In the second part of the MAGNIMS consensus guidelines on the use of MRI in patients with MS, we focus on prognostic and monitoring applications. This report

Competing interests

The authors declare no competing interests. provides recommendations from an expert panel on how and when to use MRI for disease and treatment monitoring, how to establish prognosis, and how to assess the efficacy and safety of treatments. In addition, we discuss new, promising MRI techniques that might become clinically relevant in the near future.

\section{Methods}

In June 2011, an international panel convened in Barcelona, Spain to discuss the use of MRI in patients with MS. This meeting was held under the auspices of MAGNIMS, an intellectually independent network of European clinical research groups that have an interest in the use of MRI to study patients with MS. The panel was composed of experts in the diagnosis and management of MS, and included neuroradiologists, neurologists and statisticians from nine MAGNIMS-affiliated institutions across six different countries (Box 1). The panel met to present and discuss data from research published in English, and to consider the recommendations contained in previous papers related to the use of MRI in patients with MS.

After the meeting, the panel set out to create specific and up-to-date recommendations for the implementation (planning, performance and interpretation) of brain and spinal cord MRI in the diagnostic process for patients with suspected MS. ${ }^{10}$ For this companion piece, the panel has established a similar set of recommendations on the use of MRI to monitor MS disease activity and establish disease 


\section{Box 1 | The MAGNIMS network}

The authors are members of the MAGNIMS (Magnetic Resonance Imaging in MS; http://www.magnims.eu/) network, a group of European clinicians and scientists with an interest in collaborative studies using MRI methods in patients with multiple sclerosis. The network is independent of any other organization and is run by a steering committee whose members are:

- Nicola de Stefano (co-chair), Department of Neurological and Behavioural Sciences, University of Siena, Italy

- Àlex Rovira (co-chair), Magnetic Resonance Unit, Hospital Vall d'Hebron, Barcelona, Spain

- Frederik Barkhof, Department of Neuroradiology, VU University Medical Centre, Amsterdam, Netherlands

- Olga Ciccarelli, Institute of Neurology, Queen Square, University College London, UK

- Christian Enzinger, Department of Neurology, Medical University Graz, Graz, Austria

- Massimo Filippi, Department of Neurology, Scientific Institute and University, Ospedale San Raffaele, Milan, Italy

- Jette Frederiksen, Department of Neurology, University of Copenhagen, Glostrup Hospital, Denmark

- Claudio Gasperini, Department of Neuroscience, Ospedale San Camillo Forlanini, Rome, Italy

- Ludwig Kappos, Department of Neurology, University Hospital, Kantonsspital, Basel, Switzerland

- Jacqueline Palace, Centre for Functional Magnetic Resonance Imaging of the Brain, University of Oxford, UK

- Maria A. Rocca, Department of Neurology, Scientific Institute and University, Ospedale San Raffaele, Milan, Italy

- Jaume Sastre-Garriga, Department of Neurology/Neuroimmunology, Hospital Vall d'Hebron, Barcelona, Spain

- Hugo Vrenken, Department of Neurology, VU University Medical Centre, Amsterdam, Netherlands

- Tarek A. Yousry, Institute of Neurology, Queen Square, University College London, UK

Permission obtained from Nature Publishing Group @ Rovira, À. et al. Nat. Rev. Neurol. 11 471-482 (2015).
The number of T2 lesions in patients with CIS has also been associated with disability accumulation, as measured by the Expanded Disability Status Scale (EDSS)..$^{13}$ However, subsequent research indicated that the topography of the lesions, in addition to the total number, has prognostic value in patients with CIS. Infratentorial lesions are of particular importance: the presence of at least one cerebellar lesion is related to an elevated conversion rate to definite MS, and the presence of at least one brainstem lesion is also associated with a higher risk of conversion, as well as increased disability accumulation. ${ }^{15}$ The relevance of infratentorial lesions in relation to clinical outcome was further underscored in a study showing that spinal cord lesions, infratentorial lesions and contrast-enhancing lesions in patients with optic neuritis have predictive value for disability accumulation at 6-year follow-up. ${ }^{16}$ Furthermore, the presence of at least two infratentorial lesions in patients presenting with CIS seems to have high predictive value for long-term disability. ${ }^{17}$

\section{MRI monitoring of disease course}

Several guidelines have tried to define the indications for and frequency of serial MRI in adults and children with an established diagnosis of MS. ${ }^{18-20}$ In general, the recommendation is that patients should be further evaluated with MRI after each unexpected clinical presentation that might be related to MS (such as unexplained or atypical symptoms of disease activity), or is not typical of MS (for example, suspected comorbidity such as vascular or neoplastic disease, or adverse effects of treatments). Treated patients with MS are a heterogeneous population with different levels of disease activity and susceptibility to drug-related adverse events. Follow-up MRI can reveal multiple measures of MS pathology, but the usefulness and reliability of these measures vary.

\section{Focal lesions}

Brain MRI is very sensitive for monitoring of disease activity and treatment efficacy in patients with MS, and parameters related to image acquisition (for example, pulse sequences, spatial resolution and MRI hardware) are relatively easy to standardize, particularly in a singlecentre setting. MRI of the spinal cord is less sensitive than brain MRI for detecting disease activity, particularly with regard to contrast-enhancing lesions. ${ }^{21,22}$ This limitation arises from the technical challenges of spinal cord MRI acquisition - relating mostly to image artefacts associated with vascular and cerebrospinal fluid pulsation - and the difficulty of standardizing the assessment of lesion count and lesion volume. In addition, most spinal cord lesions are clinically symptomatic, and a strong relationship exists between the development of new lesions in the brain and the development of new lesions in the spinal cord. ${ }^{23}$ Taken together, these issues indicate that serial spinal cord imaging for the detection of new focal lesions might add little to brain imaging for monitoring of disease activity and progression. Thus, the relevance of spinal cord imaging for routine follow-up seems rather limited. 

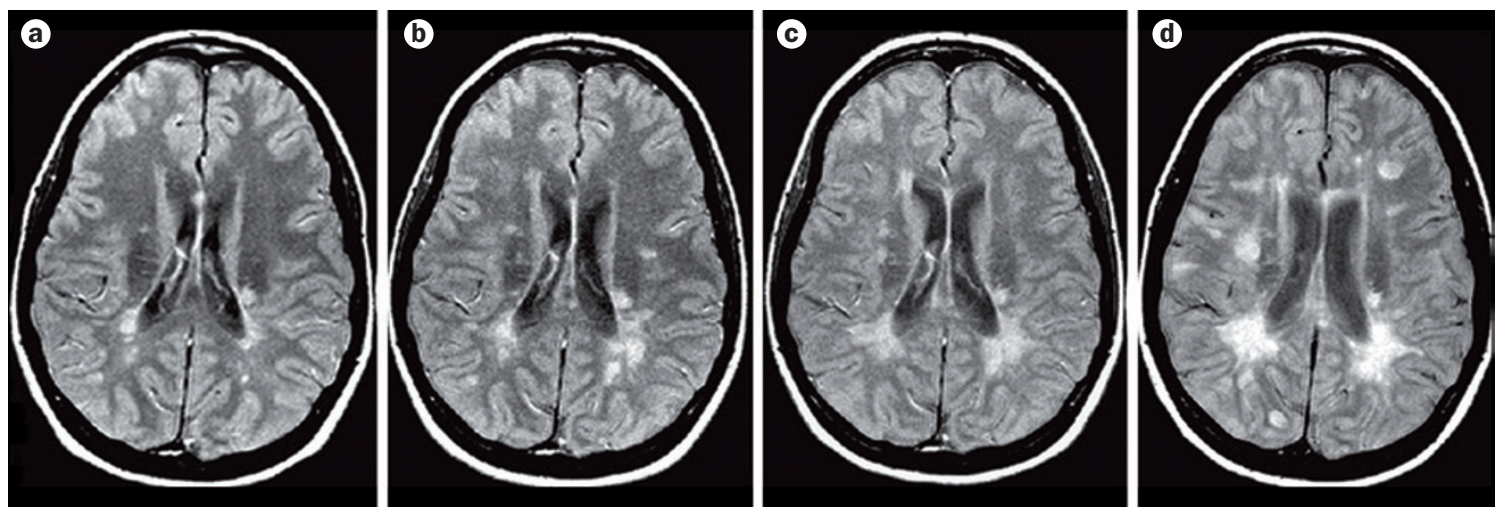

Figure 1 | Serial MRI in a patient with relapsing-remitting multiple sclerosis. Proton-density weighted MRI scans obtained at $\mathbf{a} \mid$ baseline, and $\mathbf{b} \mid 1$ year, $\mathbf{c} \mid 2$ years and $\mathbf{d} \mid 3$ years later. Disease progression can clearly be seen in the form of new and enlarging focal lesions over time, shown here as hyperintensities (white spots).

Ideally, brain MRI should be performed on the same MRI system and using the same imaging protocol-that is, the same pulse sequences and spatial resolution - as the reference (baseline) scan. Contrast-enhanced T1-weighted sequences are recommended to detect acute inflammation. However, depending on the clinical situation and the scan interval, demonstration of active (new or enlarging) T2 lesions can deliver sufficient information about subclinical disease activity and disease progression (Figure 1). ${ }^{24,25}$ In addition to contrast-enhancing and active T2 lesions to measure acute MS-related inflammation, several MRI markers of focal neurodegeneration should be considered, such as chronic T1 hypointense lesions ('black holes') that persist longer than 6 months. ${ }^{26-29}$ This imaging finding may hold promise for predicting disability progression and monitoring remyelination, and represents a possible new outcome marker for MS therapies. ${ }^{30,31}$

\section{Global brain volume changes}

The pathological hallmark of MS is the presence of multiple focal demyelinating lesions in the cerebral white and grey matter, but substantial brain atrophy can also occur. ${ }^{32}$ Over the past few years, several studies have used MRIderived methods to assess brain volume changes, revealing that atrophy can be present even in the early stages of MS, and that it advances over the disease course (Figure 2). ${ }^{33,34}$ Generally speaking, brain volume changes can be an important measure of tissue damage in patients with MS. ${ }^{32}$ Indeed, baseline atrophy and high rates of subsequent volume loss are associated with cognitive impairment, fatigue and disability progression over the long term. ${ }^{32,35-41}$

In a complex disease such as MS, brain volume loss results from the sum of and interactions between various destructive pathological processes ${ }^{42}$ including irreversible demyelination, and axonal and/or neuronal loss. The neurodegenerative pathology that occurs in MS is an important target for treatment; thus, MRI brain volume measures have been used in randomized clinical trials to monitor the effects of disease-modifying therapies on these parameters. ${ }^{26,32}$ In a recent meta-analysis of clinical trials, the overall effect of treatments on brain atrophy correlated with the effect on disability. ${ }^{43}$ In many trials, however, disease-modifying drugs (DMDs) have produced only moderate evidence of a reduction in brain volume loss. Indeed, anti-inflammatory drugs have been shown to excessively decrease brain volume within the first 6 months to 1 year of treatment, followed by stabilization during the second year of treatment. ${ }^{41}$ This phenomenon is called pseudoatrophy, and it seems to be directly associated with the resolution of ongoing white matter inflammation induced at the time of treatment initiation. ${ }^{32,44-47}$ To identify pseudoatrophy during a clinical trial, brain volume should be measured every 3-6 months. ${ }^{25}$

In addition to disease-specific changes, lifestyle-related factors (including alcohol consumption, smoking, dehydration and BMI), genetics (such as the presence of an $A P O E^{*} \varepsilon 4$ allele), and concomitant pathophysiological conditions (such as diabetes and/or other cardiovascular risk factors) can affect brain volume. Clinical interpretation of brain volume loss in patients with MS can be difficult in the context of these other factors. ${ }^{48}$ Moreover, differences in the quality and capabilities of MRI hardware, and in the software packages used for analysis or processing, can generate notable variability in brain atrophy assessments. ${ }^{32,49}$

For the above reasons, caution must be exercised when interpreting apparent changes in the rate of brain volume loss. We believe that the use of longitudinal brain volume assessment as a marker of disease progression in individual patients cannot be considered to be reliable at present. Further studies are needed to establish normative values for brain volume changes-both in healthy individuals and in patients with MS - that take the various potential confounding factors into account.

\section{The role of advanced and quantitative MRI}

Quantitative MRI techniques, including magnetization transfer ${ }^{50}$ and diffusion tensor imaging (DTI), ${ }^{51}$ can measure the extent of structural changes that occur within and outside focal lesions in white and grey matter. Moreover, these techniques can characterize the pathological nature of these changes, as has been shown by correlative histopathological-MRI studies.

The magnetization transfer ratio provides a quantitative estimate of the capacity of protons that are bound to the brain tissue matrix to exchange magnetization with 

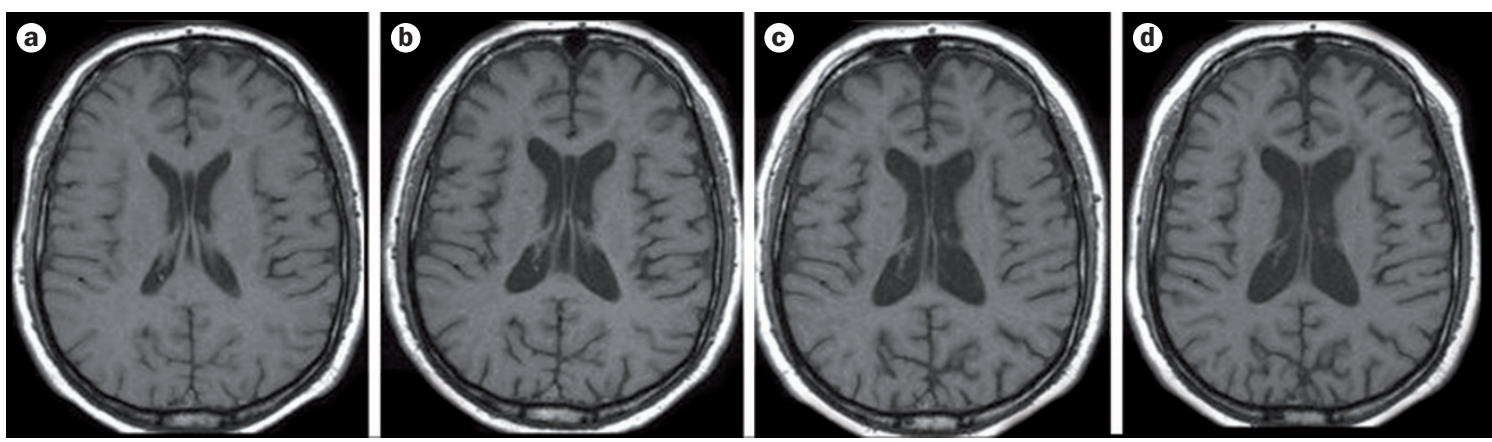

Figure 2 | Serial T1-weighted MRI scans in a patient with multiple sclerosis. a | Baseline scan. b-d | Regular scans over a 6 -year follow-up period. Disease progression can be seen in the form of the increasing size of ventricular and subarachnoid spaces. These changes reflect brain volume loss over time, indicating progressive neurodegeneration.

the surrounding free water. Decreases in the magnetization transfer ratio have been shown to correlate with the degree of myelin loss and axonal damage in patients with MS..$^{52,53}$

DTI is sensitive to the orientation and density of cellular structures that hinder water diffusion. The local tissue microstructure is evaluated with several indices, including mean diffusivity and fractional anisotropy, which correlate with myelin content, tissue integrity and axonal loss. ${ }^{54}$ Proton magnetic resonance spectroscopy $\left({ }^{1} \mathrm{H}-\mathrm{MRS}\right)$ can add information on the biochemical nature of MS-related abnormalities, by quantifying several CNS metabolites. ${ }^{55} \mathrm{~T} 2$ hypointense areas and reduced $\mathrm{T} 2{ }^{*}$ relaxation time (or its reciprocal $\mathrm{R} 2^{\star}$ ) are thought to be associated with iron deposition, which is believed to be a sign of neurodegeneration in patients with MS. ${ }^{56}$

Application of these techniques to characterize the extent and distribution of MS-related damage within focal lesions or in normal-appearing white and grey matter has shown that tissue disruption in patients with progressive disease is more severe and more widely distributed than in patients with relapsing forms of MS. ${ }^{57}$ Additionally, structural CNS damage has been shown to progress at different rates across the major clinical phenotypes of MS. Global and regional quantitative MRI abnormalities correlate with the severity of clinical and cognitive impairment, and advanced and quantitative MRI techniques seem to be useful for predicting subsequent accumulation of clinical disability and cognitive impairment..$^{57,58}$

Quantitative MRI techniques might enable measurement and monitoring of disease-related mechanisms that occur before the development of atrophy, which primarily occurs in the late stages of MS. To date, very few clinical trials have included these metrics as outcome measures. ${ }^{59-62}$ One method that was developed to monitor changes in the magnetization transfer ratio in individual lesion voxels revealed evidence consistent with demyelination and remyelination within the same lesion. Of note, signs of remyelination were still present in some lesions 3 years after their formation. ${ }^{63}$ The potential of quantitative MRI methods was demonstrated in a single-centre clinical trial that used magnetization transfer MRI, which suggested that alemtuzumab protects against grey matter damage. ${ }^{64}$ A recent combined magnetization transfer MRI and ${ }^{1} \mathrm{H}$-MRS study showed that patients treated with laquinimod tend to accumulate less microscopic white and grey matter damage than those receiving placebo. ${ }^{65}$

Despite these promising results, the actual contribution of advanced MRI techniques to clinical management has not been fully validated, especially in a longitudinal manner. Furthermore, their use for monitoring treatment effects is hampered by a lack of standardization between centres. ${ }^{58}$

\section{Statements and recommendations}

- T2-weighted and contrast-enhanced T1-weighted brain MRI are the modalities of choice for MS disease monitoring, revealing acute and active inflammation, and clinically silent disease progression ${ }^{24,25}$

- The use of spinal cord MRI in addition to brain MRI is not recommended for routine monitoring (in contrast to MS diagnosis), and should be limited to certain clinical situations (such as unexplained and/or unexpected spinal cord symptoms $)^{21-23}$

- Assessment of brain volume does not have a role in the diagnostic process of MS, but can be a good predictor of long-term disability ${ }^{32}$

- Measures of brain volume can be used in clinical studies and as end points in clinical trials, but confounding factors and pseudoatrophy should be taken into account ${ }^{44-47}$

- Rates of change in brain volume are not recommended as a marker of disease progression in individual patients, owing to the technical, biological and pharmacological factors that can influence the measurement and interpretation of atrophy rate $e^{48}$

- The use of advanced MRI methods for MS disease monitoring is promising but has not been well investigated; their value is potentially limited by a lack of standardization, and advanced MRI is, therefore, not recommended for routine clinical use $\mathrm{e}^{57}$

\section{Evaluating response to treatment}

Patients with MS who continue to experience clinical and/or MRI-visible disease activity despite treatment with DMDs are categorized as 'nonresponders. ${ }^{\text {'6-69 }}$ Early identification of nonresponders to first-line therapies would enable a prompt switch to a more effective treatment,${ }^{70}$ but predicting which individual patients will respond to DMDs, and to what degree, is challenging. 

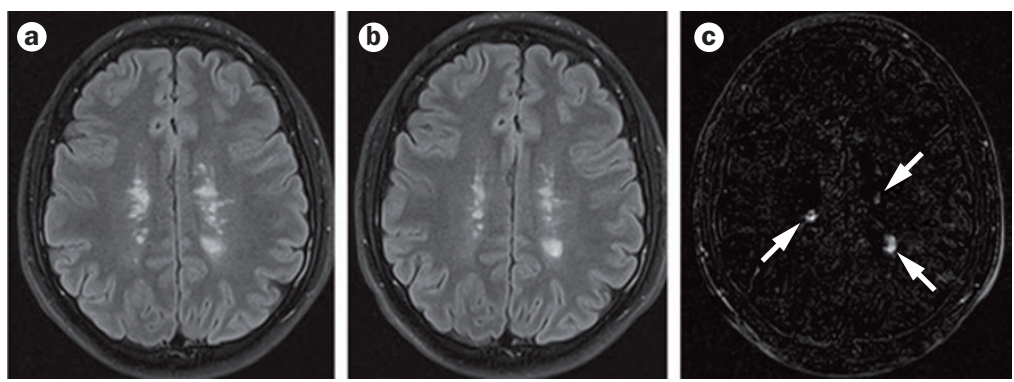

Figure $\mathbf{3}$ | Subtraction MRI in a patient with relapsing-remitting multiple sclerosis. T2 fluid-attenuated inversion recovery (FLAIR) images $\mathbf{a} \mid$ at baseline and $\mathbf{b} \mid$ after 1 year. c $\mid$ A subtraction of these two images highlights the new and enlarging lesions (arrows). not be taken into consideration. Furthermore, some drugs, such as glatiramer acetate, require up to 6 months to become effective. ${ }^{81,83}$ Therefore, the presence of new T2 lesions on a 6-12-month follow-up scan does not necessarily reflect suboptimal response; it could simply be ongoing disease activity during the period before treatment was initiated or before the drug became effective. ${ }^{84,85}$ Accordingly, some experts have proposed that the reference scan should be performed 6 months after-rather than before-treatment initiation..$^{81,86}$

\section{Follow-up measurement}

Gadolinium-enhancing lesions are typically easier to identify than new and/or enlarged T2 lesions, and the process is also less dependent on technical factors such as scan repositioning. Furthermore, some new T2 lesions can only be visually detected after being identified as new gadolinium-enhancing lesions, owing to their small size or their location in areas with confluent lesions. ${ }^{87}$ Nonetheless, recognition of disease activity cannot rely exclusively on gadolinium-enhancing lesions. New inflammatory lesions take up gadolinium for only around 3 weeks after development, ${ }^{88}$ and the recommended interval between baseline and follow-up scans is typically 3-6 months. Therefore, contrast-enhancing lesions are not sufficiently sensitive to act as sole measures of disease activity.

Detection of active T2 lesions can be hindered by multiple factors, including a high load of inactive T2 lesions, inadequate repositioning of serial scans, and interobserver variability ${ }^{89}$ Image subtraction can overcome these issues, thus providing good visualization and quantification of active and negatively active (that is, shrunken or resolved) T2 lesions (Figure 3). ${ }^{90}$ However, subtraction requires time-consuming post-processing steps, and is susceptible to artefacts. Long-interval T2-weighted sequences can be processed with automated subtraction, which has been used in a multicentre trial to provide greater power for assessing treatment efficacy than is possible with monthly contrast-enhanced T1-weighted imaging. ${ }^{90,91}$ Application of automated subtraction in treatment trials or for treatment monitoring can improve cost-effectiveness and lower the risk of adverse effects associated with repeated contrast administration. Recent data have shown that automated identification of new and/or enlarged T2 lesions is robust, accurate and sensitive, thus supporting its use for evaluating treatment efficacy in clinical trials. ${ }^{92}$

Nonetheless, additional work is needed before these methods can be incorporated into clinical practice to assess MS activity. Proposed scoring methods to identify patients with a suboptimal treatment response on the basis of combined clinical and radiological measures at follow-up have shown considerable variation (Table 1). Moreover, these criteria have been developed almost exclusively in patients receiving different formulations of IFN- $\beta$; few data are available from patients undergoing treatment with other DMDs in clinical practice. Future MRI criteria for predicting treatment response should incorporate new imaging measures (for example, brain atrophy or spinal cord pathology), genetic factors and 
Table 1 | MRI criteria for predicting treatment response

\begin{tabular}{|c|c|c|}
\hline Criteria & Outcome measure & Results \\
\hline $\begin{array}{l}\text { Three or more active lesions } \\
\text { in } 1 \text { year }{ }^{134}\end{array}$ & $\begin{array}{l}\text { Disability progression over } \\
3 \text { years }\end{array}$ & $\begin{array}{l}\text { OR } 8.3 \\
71 \% \text { sensitivity } \\
71 \% \text { specificity }\end{array}$ \\
\hline $\begin{array}{l}\text { Three or more active lesions plus } \\
\text { one or more relapse or } \geq 1 \text { point } \\
\text { confirmed EDSS score increase } \\
\text { in } 1 \text { year }{ }^{67}\end{array}$ & $\begin{array}{l}\text { Relapse rates and/or disability } \\
\text { progression over } 3 \text { years }\end{array}$ & $\begin{array}{l}\text { OR 3.3-9.8 } \\
\text { for relapses } \\
\text { OR } 6.5-7.1 \\
\text { for progression }\end{array}$ \\
\hline $\begin{array}{l}\text { Modified Rio Score } \geq 2 \text { and more } \\
\text { than five new } T 2 \text { lesions plus one } \\
\text { relapse; or more than one relapse }\end{array}$ & $\begin{array}{l}\text { Relapse rates and/or disability } \\
\text { progression over } 4 \text { years }\end{array}$ & $\begin{array}{l}24 \% \text { sensitivity } \\
97 \% \text { specificity }\end{array}$ \\
\hline $\begin{array}{l}\text { One or more relapse and nine or } \\
\text { more } T 2 \text { lesions or a minimum of } \\
\text { one } C E L^{80}\end{array}$ & $\begin{array}{l}\text { Relapse rates and/or disability } \\
\text { progression over } 4 \text { years }\end{array}$ & $\begin{array}{l}34 \% \text { sensitivity } \\
90 \% \text { specificity }\end{array}$ \\
\hline $\begin{array}{l}\text { One or more relapse, or at least } \\
\text { one } \mathrm{CEL}^{80}\end{array}$ & $\begin{array}{l}\text { Relapse rates and/or disability } \\
\text { progression over } 4 \text { years }\end{array}$ & $\begin{array}{l}68 \% \text { sensitivity } \\
80 \% \text { specificity }\end{array}$ \\
\hline $\begin{array}{l}\text { One or more CELs, or at least two } \\
\text { new T2 lesions }{ }^{80}\end{array}$ & $\begin{array}{l}\text { Relapse rates and/or disability } \\
\text { progression over } 4 \text { years }\end{array}$ & $\begin{array}{l}61 \% \text { sensitivity } \\
83 \% \text { specificity }\end{array}$ \\
\hline
\end{tabular}

laboratory biomarkers to enrich the predictive power for treatment response in individual patients, and should be validated in patients receiving DMDs other than IFN- $\beta$.

\section{Statements and recommendations}

- Baseline (pretreatment) brain MRI measures do not satisfactorily predict treatment response in clinical practice, ${ }^{70}$ but scans within the first few months of treatment initiation can predict treatment response in patients receiving first-line $\mathrm{DMDs}^{67,79-82}$

- Follow-up brain MRI, including T2-weighted and contrast-enhanced T1-weighted sequences, should be performed 12 months after starting treatment and compared with a reference scan obtained after the treatment has taken effect $^{69}$

- Timing of this reference scan should consider the precise time that treatment was started and the drug's mechanism of action; scans at 6 months after the start of treatment should be considered ${ }^{69}$

- New T2 lesion count requires high-quality, comparable MRI scans, and must be interpreted by highly qualified, trained readers to minimize observer variability ${ }^{89}$

- MRI subtraction facilitates recognition of changes in focal lesions over time, thereby increasing the power of serial imaging ${ }^{11}$

- Automated subtraction improves accuracy and sensitivity for identifying new and/or enlarged T2 lesions, although validation studies and technical improvements are required before this strategy can be incorporated into clinical practice ${ }^{92}$

- The available data do not suffice to support the use of brain volume or spinal cord measures for predicting treatment response in individual patients

\section{Detection of adverse effects}

The role of MRI in MS drug surveillance is becoming more important as the new generation of immunomodulatory and immunosuppressive drugs enter more widespread use. In general, MRI has three major tasks in this context: detection of persistent disease activity, comorbidities (such as vascular or neoplastic disorders) and adverse effects (including opportunistic infections). ${ }^{7,93}$

The crucial role of MRI in pharmacovigilance is made evident by the case of natalizumab, a recombinant humanized monoclonal antibody against a4-integrin. ${ }^{94}$ This treatment for MS is highly effective, but is associated with progressive multifocal leukoencephalopathy (PML), a potentially life-threatening adverse effect. Imaging findings of natalizumab-associated PML are heterogeneous and can, therefore, be difficult to interpret. However, experienced readers who are fully informed of patient backgrounds can reliably detect natalizumab-associated PML via MRI, ${ }^{95-97}$ even before patients manifest symptoms. ${ }^{98}$ Detection of PML lesions at this asymptomatic or presymptomatic stage is associated with improved survival and functional outcome. ${ }^{99}$

Up to now, there have been no strict guidelines on how and when to perform MRI for safety monitoring in natalizumab-treated patients with MS. Factors such as lengthy treatment duration, past use of other immunosuppressive drugs, and the presence (and levels) of antibodies against the JC virus (JCV) have been associated with an increased risk of PML in these patients. ${ }^{100-103}$ Therefore, the frequency of MRI scanning should be adjusted according to the individual's risk of PML.

Substantial evidence indicates that T2-FLAIR (fluidattenuated inversion recovery) is the most sensitive sequence for detecting PML. ${ }^{104}$ Diffusion-weighted imaging is highly sensitive for depicting acute demyelination, and can also aid differentiation of acute PML lesions from chronic and subacute demyelinating MS lesions. ${ }^{104}$ Therefore, frequent MRI scanning using T2-FLAIR and diffusion-weighted sequences in combination with conventional T2-weighted images is recommended for screening patients at high risk of developing PML. In patients with MRI lesions suggestive of PML, the MRI protocol should be extended to include contrastenhanced T1-weighted imaging to detect inflammatory features and the possible coincidence of PML and PMLimmune reconstitution inflammatory syndrome (IRIS), particularly during follow-up..$^{95,105}$

MRI-based monitoring for early PML detection is appropriate not only for patients taking natalizumab, but also for other DMDs, including alemtuzumab, ${ }^{106}$ rituximab ${ }^{107}$ and dimethyl fumarate. ${ }^{108-110}$

The value of MRI for treatment monitoring goes beyond PML detection. Other opportunistic infections leading to encephalitis (such as varicella zoster) can also develop in patients with MS, as has been shown during or after treatment with fingolimod, a sphingosine-1-phosphate receptor modulator approved for MS treatment. ${ }^{11-114}$ In addition, serious paradoxical reactions, such as tumefactive demyelination or overwhelming inflammatory demyelination, can occur during fingolimod treatment. ${ }^{115,116}$

Given the growing number of immunosuppressive and immunomodulatory treatments for MS, MRI-based safety monitoring will become increasingly complex, as 


\section{Box 2 | Protocol for follow-up MRI in patients with MS}

Regular brain MRI scans are essential for monitoring disease progression in patients with MS, but-in contrast to the diagnosis of MS—spinal imaging is not necessary for most patients. The frequency and make-up of each follow-up is determined by the needs of the individual patient.

\section{Recommendations for routine follow-up}

- Contrast-enhanced T1-weighted scans and T2-weighted scans can reveal inflammation and the development of new and/or enlarging lesions

- MRI subtraction techniques can facilitate the detection of new lesions across serial scans, but automated subtraction should be used with caution

- T2-weighted images, T2 fluid-attenuated inversion recovery (FLAIR) and diffusion-weighted imaging should also be used in patients at risk of serious treatment-related adverse effects, such as PML

- Follow-up scans should be conducted at least annually, and as often as every 3-4 months in patients who require enhanced pharmacovigilance

Recommendations for further clinical study

- Changes in total brain, grey matter and/or white matter volumes can predict disability, but these measures are difficult to obtain and interpret in the routine clinical setting, which limits their clinical relevance to standard patient care

- Magnetization transfer imaging, diffusion tensor imaging and proton magnetic resonance spectroscopy show promise for uncovering the mechanisms of MS pathogenesis, but these findings require further validation to confirm their clinical value

- As the availability of new MRI hardware (for example, $7 \mathrm{~T} \mathrm{MRI)} \mathrm{increases,}$ scanning protocols may need to be updated

Abbreviations: MS, multiple sclerosis; PML, progressive multifocal leukoencephalopathy. well as more valuable. An example of this complexity can be seen in patients treated with natalizumab who switch to different drugs, such as fingolimod or alemtuzumab, owing to drug safety concerns. Evidence is accumulating that drug-related adverse effects can occur at the time an MS treatment is discontinued or even several months after a new treatment is started (so-called 'carryover opportunistic infections'). ${ }^{117-119}$ Therefore, strict pharmacovigilance, including frequent MRI scanning, should be performed in patients who switch therapies, so as to detect resurgent MS disease activity and adverse effects such as opportunistic infections.

\section{Statements and recommendations}

- MRI should be included in drug surveillance programmes to screen for opportunistic infections, ${ }^{103,114}$ unexpected disease activity (including paradoxical reactions), ${ }^{82,115,116}$ and comorbidities ${ }^{7,93}$

- For natalizumab-treated patients with MS who are at high risk of PML (JCV seropositive, treatment duration $\geq 18$ months), we recommend brain MRI screening every 3-4 months using an MRI protocol that includes FLAIR, T2-weighted and diffusion-weighted imaging ${ }^{95-99,101,104}$

- In patients at low risk of PML (JCV seronegative), we recommend brain MRI assessment once a year using the same MRI protocol ${ }^{95-99,101,104}$

- In patients at high risk of developing opportunistic infections who are switching DMDs, we recommend brain MRI at the time that the current treatment is discontinued and after the new treatment is started ${ }^{117-119}$

- Enhanced pharmacovigilance, including brain MRI every 3-4 months for up to 12 months, is required in patients who switch from natalizumab to other therapeutics (including fingolimod, alemtuzumab and dimethyl fumarate) $)^{117-119}$

\section{Standardized follow-up MRI protocol}

The use of MRI in the routine follow-up of patients with MS is less straightforward than in the diagnostic process, owing largely to the experimental nature of many of the techniques that have been used to measure disease progression. Here, we present a brief recommendation for a standard approach to patient monitoring, which is based on MRI techniques that have high clinical relevance (Box 2). These guidelines will require revisions as the use of advanced MRI techniques increases, and the availability of high-field-strength MRI widens.

Although follow-up MRI scans should be as consistent as possible with baseline or reference scans, fewer sequences are necessary than we have recommended for diagnosis. ${ }^{10}$ The specific follow-up protocol strongly depends on the purpose of the scan (for example, treatment efficacy monitoring versus PML screening). To detect new or enlarging lesions, proton-density and/or T2-FLAIR and T2-weighted fast or turbo spin-echo sequences should be used. A gadolinium-enhanced T1-weighted sequence can increase confidence in the detection of lesions with high inflammatory activity. As with diagnostic scans, the delay between contrast adminstration and T1 acquisition-a minimum of $5 \mathrm{~min}$ - can provide an opportunity to perform proton density-weighted, T2-weighted and/or T2-FLAIR after contrast administration and before the $\mathrm{T} 1$ postcontrast acquisition. This approach optimizes the total scanning time. Diffusion-weighted scans should also be considered in patients at risk of PML.

Follow-up MRI should be conducted at least once every year in patients with MS, but patients at risk of serious treatment-related adverse events may need to be monitored more frequently, for example, every 3-4 months. Accurate positioning of follow-up and reference scans is essential for the accurate assessment of changes in lesion size and number over time. Algorithms that automatically position serial MRI scans are currently difficult to implement in routine clinical use, but might be useful in the near future.

All scans should be performed at a field strength of at least $1.5 \mathrm{~T}$, though higher field strengths might reveal more new lesions. For 2D sequences, slice thickness should be no more than $3 \mathrm{~mm}$ with an in-plane spatial resolution of $1 \times 1 \mathrm{~mm}$ (voxel size $3 \times 1 \times 1 \mathrm{~mm}$ ). Voxels in $3 \mathrm{D}$ sequences should be $1 \mathrm{~mm}^{3}$. Further technical details for the above sequences can be found in the first part of our consensus guidelines. ${ }^{10}$

\section{Statements and recommendations}

- Follow-up MRI scans typically require fewer sequences per session than do diagnostic scans, and can be completed in 20-25 min

- Routine monitoring should be conducted every 3-12 months, depending on patient characteristics such as disease duration, comorbidities and current treatment 
- Several advanced techniques show promise for investigating MS pathology, and may need to be incorporated into future protocols

\section{Future perspectives}

The role of MRI in MS disease monitoring is gaining research interest as well as clinical importance. Treatment options and strategies for patients with MS are dynamically moving towards an individualized approach that includes conventional targets-immune modulation and immune suppression-and new targets such as neuroprotection and remyelination. ${ }^{26,31,120}$ Therefore, we will need new MRI biomarkers that focus on additional and alternative aspects of MS pathology.

A promising source for a new biomarker is grey matter pathology, as correlations between cortical lesions and important clinical outcome measures, such as cognition, are stronger when grey matter and white matter are evaluated jointly. ${ }^{121-126}$ Several MRI techniques, including double inversion recovery and phase-sensitive inversion recovery, have been used to detect, score and interpret cortical grey matter lesions, but these applications lack standardization. ${ }^{127-129}$ Advanced, quantitative imaging techniques may also acquire a central role for evaluating the course of MS pathology in the near future. Standardization of these methods, particularly in multicentre settings, will be a challenge.

The use of MRI in the context of disease and treatment monitoring might benefit from a paradigm shift away from focal inflammatory lesions and whole-brain atrophy and towards certain clinically relevant anatomical structures, such as the thalamus, cortical grey matter and upper cervical spinal cord. ${ }^{58,65,130}$ This shift will require greater implementation of new-generation high-field MRI systems for the detection and quantification of MS pathology, which have been investigated in relation to the diagnosis and differential diagnosis of MS. ${ }^{131-133}$ Whether high-field MRI technology might also be of value for
MS disease monitoring must be further evaluated, but it seems likely that these techniques, along with the new MRI markers they reveal, will have an important impact on MS disease monitoring in the future.

\section{Future needs and recommendations}

- Future research must identify new MRI markers of neuroinflammation and neuroprotection, particularly in the context of grey matter pathology (cortical and deep grey matter structures), remyelination and neuronal repair

- These new markers might require next-generation MRI technology, including new advanced pulse sequences, and enhanced hardware, such as new coils, multitransmit techniques and ultra-high field strengths

- Increased efforts are needed to implement and harmonize various advanced MRI techniques, and to standardize the acquisition and interpretation of MRI in patients with MS

- Systematic research is needed to assess the added value of alternative versus standard pulse sequences, MRI subtraction techniques and serial MRI scanning for disease and safety monitoring (including the most cost-effective follow-up frequency)

\section{Conclusions}

This Expert Consensus Document discusses the contribution of MRI to the monitoring of MS disease and treatment. The guidelines and recommendations provided are intended to aid decision-making regarding the MRI protocol and timing of follow-up scans, and the use of additional MRI techniques for prognostication and monitoring of patients with MS. Although this paper is based on the most recent data and our extensive clinical experience with MS treatments, we note that care for patients with MS is constantly influenced by new treatment strategies and new imaging approaches. Therefore, these guidelines should be periodically updated.
1. Fazekas, F. et al. The contribution of magnetic resonance imaging to the diagnosis of multiple sclerosis. Neurology 53, 448-456 (1999).

2. Miller, D. H. et al. Role of magnetic resonance imaging within diagnostic criteria for multiple sclerosis. Ann. Neurol. 56, 273-278 (2004).

3. Charil, A. et al. MRI and the diagnosis of multiple sclerosis: expanding the concept of "no better explanation". Lancet Neurol. 5, 841-852 (2006).

4. McDonald, W. I. et al. Recommended diagnostic criteria for multiple sclerosis: guidelines from the International Panel on the diagnosis of multiple sclerosis. Ann. Neurol. 50, 121-127 (2001).

5. Polman, C. H. et al. Diagnostic criteria for multiple sclerosis: 2005 revisions to the "McDonald Criteria”. Ann. Neurol. 58, 840-846 (2005).

6. Polman, C. H. et al. Diagnostic criteria for multiple sclerosis: 2010 revisions to the McDonald criteria. Ann. Neurol. 69, 292-302 (2011).

7. Rommer, P. S. et al. Requirement for safety monitoring for approved multiple sclerosis therapies: an overview. Clin. Exp. Immunol. 175 , 397-407 (2014).

8. Cross, A. H. \& Naismith, R. T. Established and novel disease-modifying treatments in multiple sclerosis. J. Intern. Med. 275, 350-363 (2014).

9. Tran, J. Q. et al. Randomized phase I trials of the safety/tolerability of anti-LINGO-1 monoclonal antibody BIIB033. Neurol. Neuroimmunol. Neuroinflamm. 1, e18 (2014).

10. Rovira, A. et al. MAGNIMS consensus guidelines on the use of MRI in multiple sclerosis-clinical implementation in the diagnostic process. Nat. Rev. Neurol. 11, 471-482 (2015).

11. Barkhof, F. The clinico-radiological paradox in multiple sclerosis revisited. Curr. Opin. Neurol. 15 239-245 (2002).

12. Tintore, M. et al. Defining high, medium and low impact prognostic factors for developing multiple sclerosis. Brain 138, 1863-1874 (2015).

13. Fisniku, L. K. et al. Disability and T2 MRI lesions: a 20-year follow-up of patients with relapse onset of multiple sclerosis. Brain 131, 808-817 (2008).

14. Swanton, J. K. et al. Early MRI in optic neuritis: the risk for clinically definite multiple sclerosis. Mult. Scler. 16, 156-165 (2010).

15. Tintore, M. et al. Brainstem lesions in clinically isolated syndromes. Neurology 75, 1933-1938 (2010).
16. Swanton, J. K. et al. Early MRI in optic neuritis: the risk for disability. Neurology 72, 542-550 (2009).

17. Minneboo, A. et al. Infratentorial lesions predict long-term disability in patients with initial findings suggestive of multiple sclerosis. Arch. Neurol. 61 217-221 (2004)

18. Simon, J. H. et al. Standardized MR imaging protocol for multiple sclerosis: Consortium of MS Centers consensus guidelines. AJNR Am. J. Neuroradiol. 27, 455-461 (2006).

19. Cook, S. D. et al. Use of magnetic resonance imaging as well as clinical disease activity in the clinical classification of multiple sclerosis and assessment of its course: a report from an international CMSC Consensus Conference, March 5-7, 2010. Int. J. MS Care 14, 105-114 (2012).

20. Verhey, L. H., Narayanan, S. \& Banwell, B. Standardized magnetic resonance imaging acquisition and reporting in pediatric multiple sclerosis. Neuroimaging Clin. N. Am. 23, 17-26 (2013).

21. Thorpe, J. W. et al. Serial gadolinium-enhanced MRI of the brain and spinal cord in early relapsing-remitting multiple sclerosis. Neurology 46, 373-378 (1996). 
22. Silver, N. C. et al. A modified protocol to improve the detection of enhancing brain and spinal cord lesions in multiple sclerosis. J. Neurol. 248, 215-224 (2001)

23. Lycklama, G. et al. Spinal-cord MRI in multiple sclerosis. Lancet Neurol. 2, 555-562 (2003).

24. Sormani, M. P. et al. Magnetic resonance active lesions as individual-level surrogate for relapses in multiple sclerosis. Mult. Scler. 17, 541-549 (2011).

25. Barkhof, F. et al. MRI monitoring of immunomodulation in relapse-onset multiple sclerosis trials. Nat. Rev. Neurol. 8, 13-21 (2011).

26. Barkhof, F., Calabresi, P. A., Miller, D. H \& Reingold, S. C. Imaging outcomes for neuroprotection and repair in multiple sclerosis trials. Imaging outcomes for neuroprotection and repair in multiple sclerosis trials. Nat. Rev. Neurol. 5, 256-266 (2009).

27. Nagtegaal, G. J. et al. Interferon beta-1b reduces black holes in a randomised trial of clinically isolated syndrome. Mult. Scler. 20, 234-242 (2014).

28. Mitjana, R. et al. Diagnostic value of brain chronic black holes on T1-weighted MR images in clinically isolated syndromes. Mult. Scler. 20 1471-1477 (2014).

29. Giorgio, A. et al. Relevance of hypointense brain MRI lesions for long-term worsening of clinical disability in relapsing multiple sclerosis. Mult. Scler. 20, 214-219 (2014).

30. Curtin, F. \& Hartung, H. P. Novel therapeutic options for multiple sclerosis. Expert Rev. Clin Pharmacol. 7, 91-104 (2014).

31. Mallik, S., Samson, R. S. Wheeler-Kingshott, C. A. \& Miller, D. H. Imaging outcomes for trials of remyelination in multiple sclerosis. J. Neurol. Neurosurg. Psychiatry 85, 1396-1404 (2014).

32. De Stefano, N. et al. Clinical relevance of brain volume measures in multiple sclerosis. CNS Drugs 28, 147-156 (2014).

33. De Stefano, N. et al. Assessing brain atrophy rates in a large population of untreated multiple sclerosis subtypes. Neurology 74, 1868-1876 (2010).

34. Fisher, E., Lee, J. C., Nakamura, K. \& Rudick, R. A. Gray matter atrophy in multiple sclerosis: a longitudinal study. Ann. Neurol. 64 255-265 (2008)

35. Popescu, V. et al. Brain atrophy and lesion load predict long term disability in multiple sclerosis. J. Neurol. Neurosurg. Psychiatry 84, 1082-1091 (2013)

36. Filippi, M. et al. Gray matter damage predicts the accumulation of disability 13 years later in MS. Neurology 81, 1759-1767 (2013)

37. Lavorgna, L. et al. Clinical and magnetic resonance imaging predictors of disease progression in multiple sclerosis: a nine-year follow-up study. Mult. Scler. 20, 220-226 (2014)

38. Gobbi, C. et al. Influence of the topography of brain damage on depression and fatigue in patients with multiple sclerosis. Mult. Scler. 20, 192-201 (2014).

39. Fisher, E. et al. Relationship between brain atrophy and disability: an 8-year follow-up study of multiple sclerosis patients. Mult. Scler. $\mathbf{6}$, 373-377 (2000)

40. Shiee, N. et al. Revisiting brain atrophy and its relationship to disability in multiple sclerosis. PLoS ONE 7, e37049 (2012).

41. Jacobsen, C. et al. Brain atrophy and disability progression in multiple sclerosis patients: a 10-year follow-up study. J. Neurol. Neurosurg. Psychiatry 85, 1109-1115 (2014).
42. Filippi, M. et al. Association between pathological and MRI findings in multiple sclerosis. Lancet Neurol. 11, 349-360 (2012).

43. Sormani, M. P., Arnold, D. L. \& De Stefano, N. Treatment effect on brain atrophy correlates with treatment effect on disability in multiple sclerosis. Ann. Neurol. 75, 43-49 (2014).

44. Zivadinov, R. et al. Mechanisms of action of disease-modifying agents and brain volume changes in multiple sclerosis. Neurology 71 , 136-144 (2008).

45. Vidal-Jordana, A. et al. Early brain pseudoatrophy while on natalizumab therapy is due to white matter volume changes. Mult. Scler. 19, 1175-1181 (2013)

46. Sastre-Garriga, J. et al. Brain atrophy in natalizumab-treated patients: a 3-year follow-up. Mult. Scler. 21, 749-756 (2015).

47. De Stefano, N. \& Arnold, D. L. Towards a better understanding of pseudoatrophy in the brain of multiple sclerosis patients. Mult. Scler. 21, 675-676 (2015).

48. Enzinger, C. et al. Risk factors for progression of brain atrophy in aging: six-year follow-up of normal subjects. Neurology 64, 1704-1711 (2005).

49. Huppertz, H. J., Kröll-Seger, J., Klöppel, S.,

Ganz, R. E. \& Kassubek, J. Intra- and interscanner variability of automated voxel-based volumetry based on a 3D probabilistic atlas of human cerebral structures. Neuroimage 49, 2216-2224 (2010).

50. Ropele, S. \& Fazekas, F. Magnetization transfer MR imaging in multiple sclerosis. Neuroimaging Clin. N. Am. 19, 27-36 (2009).

51. Rovaris, M., Agosta, F., Pagani, E. \& Filippi, M. Diffusion tensor MR imaging. Neuroimaging Clin. N. Am. 19, 37-43 (2009)

52. van Waesberghe, J. H. et al. Axonal loss in multiple sclerosis lesions: magnetic resonance imaging insights into substrates of disability. Ann. Neurol. 46, 747-754 (1999).

53. Schmierer, K., Scaravilli, F., Altmann, D. R. Barker, G. J. \& Miller, D. H. Magnetization transfer ratio and myelin in postmortem multiple sclerosis brain. Ann. Neurol. 56, 407-415 (2004).

54. Mottershead, J. P. et al. High field MRI correlates of myelin content and axonal density in multiple sclerosis-a post-mortem study of the spinal cord. J. Neurol. 250, 1293-1301 (2003).

55. Sajja, B. R., Wolinsky, J. S. \& Narayana, P. A. Proton magnetic resonance spectroscopy in multiple sclerosis. Neuroimaging Clin. N. Am. 19, 45-58 (2009).

56. Langkammer, C. et al. Quantitative MR imaging of brain iron: a postmortem validation study. Radiology 257, 455-462 (2010).

57. Filippi, M. \& Rocca, M. A. New magnetic resonance imaging biomarkers for the diagnosis of multiple sclerosis. Expert Opin. Med. Diagn. 6 , 109-120 (2012)

58. Filippi, M., Absinta, M. \& Rocca, M. A. Future MR tools in multiple sclerosis. J. Neurol. Sci. 331 14-18 (2013)

59. Inglese, M. et al. The effect of interferon $\beta-1 b$ on quantities derived from MT MRI in secondary progressive MS. Neurology 60, 853-860 (2003).

60. Filippi, M. et al. European study on intravenous immunoglobulin in multiple sclerosis: results of magnetization transfer magnetic resonance imaging analysis. Arch. Neurol. 61, 1409-1412 (2004).

61. Narayanan, S. et al. Axonal metabolic recovery in multiple sclerosis patients treated with interferon beta-1b. J. Neurol. 248, 979-986 (2001).

62. Sajja, B. R., Narayana, P. A., Wolinsky, J. S. \& Ahn, C. W. Longitudinal magnetic resonance spectroscopic imaging of primary progressive multiple sclerosis patients treated with glatiramer acetate: multicenter study. Mult. Scler. 14, 73-80 (2008).

63. Chen, J. T., Collins, D. L., Atkins, H. L., Freedman, M. S. \& Arnold, D. L. Magnetization transfer ratio evolution with demyelination and remyelination in multiple sclerosis lesions. Ann. Neurol. 63, 254-262 (2008).

64. Button, T. et al. Magnetization transfer imaging in multiple sclerosis treated with alemtuzumab. Mult. Scler. 19, 241-244 (2012).

65. Filippi, M. et al. Placebo-controlled trial of oral laquinimod in multiple sclerosis: MRI evidence of an effect on brain tissue damage. J. Neurol. Neurosurg. Psychiatry 85, 851-858 (2014).

66. Río, J. et al. Defining the response to interferon- $\beta$ in relapsing-remitting multiple sclerosis patients. Ann. Neurol. 59, 344-352 (2006).

67. Río, J. et al. Measures in the first year of therapy predict the response to interferon $\beta$ in MS. Mult. Scler. 15, 848-853 (2009).

68. Río, J., Comabella, M. \& Montalban, X. Predicting responders to therapies for multiple sclerosis. Nat. Rev. Neurol. 5, 553-560 (2009).

69. Rudick, R. A., Lee, J. C., Simon, J., Ransohoff, R. M. \& Fisher, E. Defining interferon $\beta$ response status in multiple sclerosis patients. Ann. Neurol. 56, 548-555 (2004).

70. Healy, B. C. et al. A method for evaluating treatment switching criteria in multiple sclerosis. Mult. Scler. 16, 1483-1489 (2010).

71. Barkhof, F. et al. Validation of diagnostic magnetic resonance imaging criteria for multiple sclerosis and response to interferon $\beta 1$ a. Ann. Neurol. 53 , 718-724 (2003)

72. Kappos, L. et al. Long-term subcutaneous interferon beta-1a therapy in patients with relapsing-remitting MS. Neurology 67, 944-953 (2006).

73. Romeo, M. et al. Clinical and MRI predictors of response to interferon-beta and glatirame acetate in relapsing-remitting multiple sclerosis patients. Eur. J. Neurol. 20, 1060-1067 (2013).

74. Signori, A., Schiavetti, I., Gallo, F. \& Sormani, M. P. Subgroups of multiple sclerosis patients with larger treatment benefits: a meta-analysis of randomized trials. Eur. J. Neurol. 22, 960-966 (2015).

75. Pérez-Miralles, F. et al. Clinical impact of early brain atrophy in clinically isolated syndromes. Mult. Scler. 19, 1878-1886 (2013).

76. Fisher, E. et al. Eight-year follow-up study of brain atrophy in patients with MS. Neurology 59, 1412-1420 (2002).

77. Sombekke, M. H. et al. Spinal cord lesions in patients with clinically isolated syndrome: a powerful tool in diagnosis and prognosis. Neurology 80, 69-75 (2013)

78. Sormani, M. P. \& De Stefano, N. Defining and scoring response to IFN- $\beta$ in multiple sclerosis. Nat. Rev. Neurol. 9, 504-512 (2013).

79. Sormani, M. P. et al. Scoring treatment response in patients with relapsing multiple sclerosis. Mult. Scler. 19, 605-612 (2013).

80. Prosperini, L. et al. Interferon beta failure predicted by EMA criteria or isolated MRI activity in multiple sclerosis. Mult. Scler. 20, 566-576 (2014).

81. Freedman, M. S. et al. Treatment optimization in MS: Canadian MS Working Group updated recommendations. Can. J. Neurol. Sci. 40, 307-323 (2013).

82. Stangel, M., Penner, I. K., Kallmann, B. A. Lukas, C. \& Kieseier, B. C. Towards the implementation of "no evidence of disease activity' in multiple sclerosis treatment: the multiple sclerosis decision model. Ther. Adv. Neurol. Disord. 8, 3-13 (2014). 
83. Dhib-Jalbut, S. Mechanisms of action of interferons and glatiramer acetate in multiple sclerosis. Neurology 58 (8 Suppl. 4), S3-S9 (2002).

84. Comi, G., Filippi, M. \& Wolinsky, J. S. European/Canadian multicenter, double-blind, randomized, placebo-controlled study of the effects of glatiramer acetate on magnetic resonance imaging - measured disease activity and burden in patients with relapsing multiple sclerosis. European/Canadian Glatiramer Acetate Study Group. Ann. Neurol. 49, 290-297 (2001).

85. Río, J. et al. Evaluating the response to glatiramer acetate in relapsing-remitting multiple sclerosis (RRMS) patients. Mult. Scler. 20, 1602-1608 (2014).

86. Filippi, M. et al. Guidelines from the Italian Neurological and Neuroradiological Societies for the use of magnetic resonance imaging in daily life clinical practice of multiple sclerosis patients. Neurol. Sci. 34, 2085-2093 (2013).

87. Miller, D. H., Barkhof, F. \& Nauta, J. J. Gadolinium enhancement increases the sensitivity of MRI in detecting disease activity in multiple sclerosis. Brain 116, 1077-1094 (1993).

88. Cotton, F., Weiner, H. L., Jolesz, F. A \& Guttmann, C. R. MRI contrast uptake in new lesions in relapsing-remitting MS followed at weekly intervals. Neurology 60, 640-646 (2003).

89. Erbayat Altay, E. et al. Reliability of classifying multiple sclerosis disease activity using magnetic resonance imaging in a multiple sclerosis clinic. JAMA Neurol. 70, 338-344 (2013).

90. Moraal, B. et al. Improved detection of active multiple sclerosis lesions: 3D subtraction imaging. Radiology 255, 154-163 (2010).

91. Moraal, B. et al. Long-interval T2-weighted subtraction magnetic resonance imaging: a powerful new outcome measure in multiple sclerosis trials. Ann. Neurol. 67, 667-675 (2010).

92. Battaglini, M. et al. Automated identification of brain new lesions in multiple sclerosis using subtraction images. J. Magn. Reson. Imaging 39, 1543-1549 (2014).

93. Weber, M. S. et al. Current treatment strategies for multiple sclerosis-efficacy versus neurological adverse effects. Curr. Pharm. Des. 18, 209-219 (2012).

94. Kappos, L. et al. Natalizumab treatment for multiple sclerosis: updated recommendations for patient selection and monitoring. Lancet Neurol. 10, 745-758 (2011).

95. Yousry, T. A. et al. MRI pattern in natalizumabassociated progressive multifocal leukoencephalopathy. Ann. Neurol. 72, 779-787 (2012).

96. Wattjes, M. P. et al. The chameleon of neuroinflammation: magnetic resonance imaging characteristics of natalizumab-associated progressive multifocal leukoencephalopathy. Mult. Scler. 19, 1826-1840 (2013).

97. Wattjes, M. P. \& Barkhof, F. Diagnosis of natalizumab-associated progressive multifocal leukoencephalopathy using MRI. Curr. Opin. Neurol. 27, 260-270 (2014).

98. Wattjes, M. P. et al. MRI pattern in asymptomatic natalizumab-associated PML. J. Neurol. Neurosurg. Psychiatry 86, 793-798 (2015).

99. Dong-Si, T. et al. Outcome and survival of asymptomatic PML in natalizumab-treated MS patients. Ann. Clin. Transl. Neurol. 1, 755-764 (2014).

100. Sørensen, P. S. et al. Risk stratification for progressive multifocal leukoencephalopathy in patients treated with natalizumab. Mult. Scler. 18 , 143-152 (2012).
101. Bloomgren, G. et al. Risk of natalizumabassociated progressive multifoca leukoencephalopathy. N. Engl. J. Med. 366 , 1870-1880 (2012).

102. Warnke, C. et al. Changes to anti-JCV antibody levels in a Swedish national MS cohort. J. Neurol. Neurosurg. Psychiatry 84, 1199-1205 (2013).

103. Plavina T. et al. Anti-JC virus antibody levels in serum or plasma further define risk of natalizumab-associated progressive multifocal leukoencephalopathy. Ann. Neurol. 76, 802-812 (2014).

104. Richert, N. et al. Imaging findings for PML in natalizumab-treated MS patients [oral 99]. Mult. Scler. 18 (Suppl. 4): 27-28 (2012).

105. Gheuens, S. et al. Simultaneous PML-IRIS after discontinuation of natalizumab in a patient with MS. Neurology 78, 1390-1393 (2012).

106. Waggoner, J., Martinu, T. \& Palmer, S. M. Progressive multifocal leukoencephalopathy following heightened immunosuppression after lung transplant. J. Heart Lung Transplant. 28, 395-398 (2009).

107. Clifford, D. B. et al. Rituximab-associated progressive multifocal leukoencephalopathy in rheumatoid arthritis. Arch. Neurol. 68, 1156-1164 (2011).

108. van Oosten, B. W., Killestein, J., Barkhof, F. Polman, C. H. \& Wattjes, M. P. PML in a patient treated with dimethyl fumarate from a compounding pharmacy. N. Engl. J. Med. 368 1658-1659 (2013).

109. Rosenkranz, T., Novas, M. \& Terborg, C. PML in a patient with lymphocytopenia treated with dimethyl fumarate. N. Engl. J. Med. 372, 1476-1478 (2015).

110. Nieuwkamp, D. J. et al. PML in a patient without severe lymphocytopenia receiving dimethyl fumarate. N. Engl. J. Med. 372, 1474-1476 (2015).

111. Gross, C. M., Baumgartner, A., Rauer, S. \& Stich, O. Multiple sclerosis rebound following herpes zoster infection and suspension of fingolimod. Neurology 79, 2006-2007 (2012).

112. Ratchford, J. N., Costello, K., Reich, D. S. $\&$ Calabresi, P. A. Varicella-zoster virus encephalitis and vasculopathy in a patient treated with fingolimod. Neurology 79 , 2002-2004 (2012).

113. Leypoldt, F. et al. Hemorrhaging focal encephalitis under fingolimod (FTY720) treatment: a case report. Neurology 72, 1022-1024 (2009).

114. Arvin, A. M. et al. Varicella-zoster virus infections in patients treated with fingolimod: risk assessment and consensus recommendations for management. JAMA Neurol. 72, 31-39 (2015).

115. Visser, F., Wattjes, M. P., Pouwels, P. J., Linssen, W. H. \& van Oosten, B. W. Tumefactive multiple sclerosis lesions under fingolimod treatment. Neurology 79, 2000-2003 (2012).

116. Pilz, G. et al. Tumefactive MS lesions under fingolimod: a case report and literature review. Neurology 81, 1654-1658 (2013).

117. Fine, A. J., Sorbello, A., Kortepeter, C. \& Scarazzini, L. Progressive multifoca leukoencephalopathy after natalizumab discontinuation. Ann. Neurol. 75, 108-115 (2014).

118. Wattjes, M. P. \& Killestein, J. Progressive multifocal leukoencephalopathy after natalizumab discontinuation: few and true? Ann. Neurol. 75, 462 (2014)

119. Killestein, J. et al. PML-IRIS during fingolimod diagnosed after natalizumab discontinuation. Case Rep. Neurol. Med. 2014, 307872 (2014).
120. Franklin, R. J., French-Constant, C., Edgar, J. M. $\&$ Smith, K. J. Neuroprotection and repair in multiple sclerosis. Nat. Rev. Neurol. 8, 624-634 (2012).

121. Geurts, J. J. \& Barkhof, F. Grey matter pathology in multiple sclerosis. Lancet Neurol. 7, 841-851 (2008).

122. Calabrese, M., Rinaldi, F., Grossi, P. \& Gallo, P. Cortical pathology and cognitive impairment in multiple sclerosis. Expert Rev. Neurother. 11 425-432 (2011).

123. Calabrese, M. et al. Cortical lesion load associates with progression of disability in multiple sclerosis. Brain 135, 2952-2961 (2012).

124. Muhlert, N. et al. The grey matter correlates of impaired decision-making in multiple sclerosis J. Neurol. Neurosurg. Psychiatry 86, 530-536 (2015).

125. Roosendaal, S. D. et al. Accumulation of cortical lesions in MS: relation with cognitive impairment Mult. Scler. 15, 708-714 (2009).

126. Nelson, F. et al. Intracortical lesions by $3 \mathrm{~T}$ magnetic resonance imaging and correlation with cognitive impairment in multiple sclerosis Mult. Scler. 17, 1122-1129 (2011).

127. Simon, B. et al. Improved in vivo detection of cortical lesions in multiple sclerosis using double inversion recovery MR imaging at 3 Tesla. Eur. Radiol. 20, 1675-1683 (2010).

128. Sethi, V. et al., Improved detection of cortical MS lesions with phase-sensitive inversion recovery MRI. J. Neurol. Neurosurg. Psychiatry 83 877-882 (2012).

129. Geurts, J. J. et al. Consensus recommendations for MS cortical lesion scoring using double inversion recovery MRI. Neurology 76, 418-424 (2011).

130. Daams, M. et al. Mean upper cervical cord area (MUCCA) measurement in long-standing multiple sclerosis: relation to brain findings and clinical disability. Mult. Scler. 20, 1860-1865 (2014).

131. Filippi, M. et al. Ultra-high-field MR imaging in multiple sclerosis. J. Neurol. Neurosurg. Psychiatry 85, 60-66 (2014).

132. Kilsdonk, I. D., Wattjes, M. P. \& Geurts, J. J. Ultra-high field MRI: looking through the 'macroscope'. J. Neurol. Neurosurg. Psychiatry 85, 4 (2014).

133. Kilsdonk, I. D., de Graaf, W. L., Barkhof, F. \& Wattjes, M. P. Inflammation high-field magnetic resonance imaging. Neuroimaging Clin. N. Am. 22, 135-157 (2012).

134. Río, J. et al. Relationship between MRI lesion activity and response to IFN- $\beta$ in relapsingremitting multiple sclerosis patients. Mult. Scler. 14, 479-484 (2008).

Author contributions

M.P.W. and À.R. researched data for the article, and M.P.W., À.R., M.T., C.T., M.P.S., N.d.S., M.F., M.A.R. and F.B. wrote the article. All authors made substantial contributions to discussions of the content, and helped to review and/or edit the manuscript before submission.

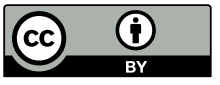

This work is licensed under a Creative Commons Attribution 4.0 International License. The mages or other third party material in this article are included in the article's Creative Commons license, unless indicated otherwise in the credit line; if the material is not included under the Creative Commons license, users will need to obtain permission from the license holder to reproduce the material. To view a copy of this license, visit http://creativecommons.org/ licenses/by/4.0/. 